EESTI NSV TEADUSTE AKADEEMIA TOIMETISED. 29. KOIDE FOOSIKA * MATEMAATIKA. 1980, NR. 1

ИЗВЕСТИЯ АКАДЕМИИ НАУК ЭСТОНСКОИ ССР. ТОМ 29 ФИЗИКА * МАТЕМАТИКА, 1980, № 1

удК 548.162 .01

T. $C A K C$

\title{
ЭЛЕКТРОННЫЕ СОСТОЯНИЯ НА ПОВЕРХНОСТИ (110) ГАЛОИДОВ ЦЕЗИЯ
}

(Представлена В. Хижняковым)

Диэлектрики в отношении электронных свойств поверхности изучались гораздо меньше, чем полупроводники. Лишь недавно впервые сделана попытка найти энергии поверхностных состояний (ПС) на поверхности (100) кристаллов $\mathrm{NaCl}$ и $\mathrm{KCl}\left[{ }^{1}\right]$. Поскольку образцы, использованные в $\left[{ }^{1}\right]$, раскалывались в воздухе, то измеренные состояния принадлежат, по-видимому, загрязненным поверхностям. Это подтверждают также чрезмерно большие значения энергии связи этих состояний, противоречащие качественным оценкам энергии собственных ПС [2]. Подобные значения энергии ПС на идеальной поверхности (110) CsBr были получены в расчете $\left[{ }^{3}\right]$. Использованные в $\left[{ }^{3}\right]$ слишком грубая модель и метод нахождения энергий ПС, по-видимому, не отражают реальные свойства поверхности ионного кристалла.

В данной работе применен метод функций Грина в модели сильной связи с учетом спин-орбитального (СО) взаимодействия и поверхностного возмущения, характерного для ионного кристалла. Рассчитанные энергии ПС на поверхности (110) кристаллов $\mathrm{CsCl}, \mathrm{CsBr}$ и CsI находятся в согласии с оценками $\left[{ }^{2}\right]$ и энергиями ПС на поверхности (100) щелочногалоидных кристаллов (ЩГК) со структурой $\mathrm{NaCl}\left[{ }^{4}\right]$.

\section{1. Электронная структура валентных зон в бесконечных кристаллах}

Из рассмотренных трех кристаллов наиболее хорошо изучен CsI, для которого имеется также несколько расчетов электронной зонной структуры $\left[{ }^{5,6}\right]$. Для $\mathrm{CsCl}$ проведен лишь один расчет $\left[{ }^{7}\right]$ энергий валентных зон в нескольких симметричных точках зоны Бриллюэна (ЗБ), а для $\mathrm{CsBr}$ имеется лишь грубая модель $\left[{ }^{3}\right]$. Поэтому здесь приведены результаты расчета зонной структуры этих кристаллов.

Использовался метод сильной связи с учетом СО эффектов в приближении Паули тем же способом, что и в $\left[{ }^{4}\right]$. Поскольку в кристаллах $\mathrm{CsCl}, \mathrm{CsBr}$ и CsI расстояние между p-зонами галоида и щелочного металла составляет всего лишь 4,$0 ; 4,5$ и 5,2 эв соответственно [ $\left.{ }^{8}\right]$, то в базис были включены, кроме $3 p$-, $4 p$ - и $5 p$-функций галоида (для $\mathrm{Cl}^{-}, \mathrm{Br}^{-}$и $\mathrm{I}^{-}$соответственно), также $5 p$-функции $\mathrm{Cs}^{+}$.

Тогда матрицы $H(\overrightarrow{\mathbf{k}})$ и $b(\overrightarrow{\mathbf{k}})$, входящие в уравнение Шредингера в k-представлении 


$$
[H(\overrightarrow{\mathbf{k}})-E(\overrightarrow{\mathbf{k}})] b(\overrightarrow{\mathbf{k}})=0
$$

имеют структуру

$$
H(\overrightarrow{\mathbf{k}})=\left|\begin{array}{cccc}
A_{1} & B_{1} & C & 0 \\
-B_{1}^{*} & A_{1}^{*} & 0 & C \\
C & 0 & A_{2} & B_{2} \\
0 & C & -B_{2}^{*} & A_{2}^{*}
\end{array}\right|, \quad b(\overrightarrow{\mathrm{k}})=\left(\begin{array}{c}
b_{1} \\
b_{2}
\end{array}\right), \quad \text { где } \quad b_{n}=\left|\begin{array}{l}
b_{x_{n}+} \\
b_{y_{n}+} \\
b_{z_{n}+} \\
b_{x_{n}-} \\
b_{y_{n}-} \\
b_{z_{n}}
\end{array}\right|
$$

Все матричные элементы $H(\overrightarrow{\mathbf{k}})$ есть матрицы 3-го порядка, «0» обозначает нулевую матрищу.

Учитывая взаимодействия до вторых соседей включительно, матричные элементы можно выразить через 10 параметров:

$$
\begin{gathered}
A_{n}=\left(\begin{array}{ccc}
A_{n, 11} & \Delta_{n} \mathrm{i} & 0 \\
-\Delta_{n} \mathrm{i} & A_{n, 22} & 0 \\
0 & 0 & A_{n, 33}
\end{array}\right), \quad C=\left(\begin{array}{lll}
C_{11} & C_{12} & C_{13} \\
C_{12} & C_{11} & C_{23} \\
C_{13} & C_{23} & C_{11}
\end{array}\right), \\
B_{n}=\Delta_{n}\left(\begin{array}{rrr}
0 & 0 & 1 \\
0 & 0 & \mathrm{i} \\
-1 & -\mathrm{i} & 0
\end{array}\right) ; \\
A_{n, 11}=(p p 0)_{n}+2(p p \sigma)_{n} \cos a k_{x}+2(p p \pi)_{n}\left(\cos a k_{y}+\cos a k_{z}\right), \\
C_{11}=(8 / 3)[(p p \sigma)+2(p p \pi)] \cos \left(a k_{x} / 2\right) \cos \left(a k_{y} / 2\right) \cos \left(a k_{z} / 2\right), \\
C_{12}=-(8 / 3)\left[(p p \sigma)-\left(p p_{\pi}\right)\right] \sin \left(a k_{x} / 2\right) \sin \left(a k_{y} / 2\right) \cos \left(a k_{z} / 2\right),
\end{gathered}
$$

$A_{n, 22}, A_{n, 33}$ и $C_{23}, C_{13}$ получаются из $A_{n, 11}$ и $C_{12}$ соответственно циклической перестановкой компонент волнового вектора $\vec{k} ;(p p \sigma),(p p \pi)-$ резонансные интегралы между ближайшими соседями; $a$ - постоянная решетки; $3 \Delta_{n}-$ величина расщепления состояний $p_{1 / 2}$ и $p_{3 / 2}$ (с учетом взаимодействия между $p$-состояниями ионов различного типа не равна энергетическому зазору между расщепленными СО взаимодействием состояниями в центре ЗБ); $n$ нумерует $p$-состояния иона галоида $(n=1)$ и $5 p$-состояния $\mathrm{Cs}^{+}(n=2)$.

Для определения параметров использовались экспериментальные значения расстояний между зонами и ширины валентных зон $\left[{ }^{8}\right]$, а также значения энергий валентных зон в симметричных точках $\Gamma, X$, $M, R$, вычисленные в $\left[{ }^{5,7}\right]$ для $\mathrm{CsCl}$ и CsI. Некоторые значения интегралов для $\mathrm{CsBr}$ были получены интерполированием параметров для $\mathrm{CsCl}$ и CsI. Во всех кристаллах ширина $5 p$-зоны $\mathrm{Cs}^{+} 3,1$ э $B$ и интегралы $(p p \sigma)_{2}=0,3032, \quad(p p \pi)_{2}=0,0425, \quad \Delta_{2}=0,570 \quad э B$. Значения ширин валентных зон $(\Delta E)$ галоидов и остальных параметров приведены в табл. 1, причем за нуль энергий выбран потолок валентной зоны галоида.

Энергетические уровни вычислялись в 43904 точках ЗБ. Поскольку ширины зон довольно значительны, обусловленная СО взаимодействием запрещенная щель в энергетическом спектре образуется лишь в $5 p$-зонах $\mathrm{Cs}^{+}$и равняется 0,$022 ; 0,067$ и 0,113 э $B$ для $\mathrm{CsCl}, \mathrm{CsBr}$ и CsI соответственно. Максимум энергий в $5 p$-зоне $\mathrm{Cs}^{+}$для всех кристаллов находится в точке $X$, а в зонах галонда и в нижней подзоне $5 p$-зоны $\mathrm{Cs}^{+}-$ 
Значения $\Delta E$ и параметров для кристаллов $\mathrm{CsCl}, \mathrm{CsBr}$ и $\mathrm{CsI}$, эB

\begin{tabular}{l|c|c|c|c|c|c|c|c}
\hline & $\Delta E$ & $(p p 0)_{1}$ & $(p p \sigma)_{1}$ & $(p p \pi)_{1}$ & $\Delta_{1}$ & $(p p 0)_{2}$ & $(p p \sigma)$ & $(p p \pi)$ \\
\hline CsCl & 1,8 & $-1,0614$ & 0,2893 & 0,020 & 0,040 & $-6,6836$ & 0,6285 & $-0,05336$ \\
CsBr & 2,1 & $-1,1218$ & 0,2891 & 0,020 & 0,160 & $-7,6427$ & 0,6246 & $-0,05303$ \\
CsI & 2,4 & $-1,1383$ & 0,2849 & $-0,015$ & 0,376 & $-8,6939$ & 0,6153 & $-0,05224$
\end{tabular}

Таблица 2

Значения энергий валентных зон в точках $\Gamma, X, R$ для кристаллов CsCl, CsBr и CsI, эB

\begin{tabular}{|c|c|c|c|c|c|c|c|c|c|c|}
\hline & $\Gamma_{8}^{-}$ & $\Gamma_{6}^{-}$ & $X-$ & $X_{6}^{-}$ & $X_{7}^{+}$ & $X_{6}^{+}$ & $R_{8}^{-}$ & $R_{6}^{-}$ & $R_{8}^{+}$ & $R_{7}^{+}$ \\
\hline $\mathrm{CsCl}$ & $\begin{array}{c}0 \\
-5,7\end{array}$ & $\begin{array}{l}-0,200 \\
-7,330\end{array}$ & $-0,443$ & $\begin{array}{l}-0,520 \\
-1,563\end{array}$ & $-5,507$ & $\begin{array}{l}-6,044 \\
-7,724\end{array}$ & $-1,680$ & $-1,8$ & $-6,890$ & $-8,600$ \\
\hline $\mathrm{CsBr}$ & $\begin{array}{c}0 \\
-6,6\end{array}$ & $\begin{array}{l}-0,528 \\
-8,262\end{array}$ & 84 & $\begin{array}{l}-0,651 \\
-1,673\end{array}$ & 6,466 & $\begin{array}{l}-7,003 \\
-8,683\end{array}$ & $-1,620$ & $-2,1$ & $-7,849$ & $-9,559$ \\
\hline CsI & $\begin{array}{c}0 \\
-7,6\end{array}$ & $\begin{array}{l}-1,146 \\
-9,292\end{array}$ & $-0,193$ & $\begin{array}{l}-0,684 \\
-2,029\end{array}$ & $-7,518$ & $\begin{array}{l}-8,054 \\
-9,734\end{array}$ & $-1,272$ & $-2,4$ & $-8,900$ & $-10,610$ \\
\hline
\end{tabular}

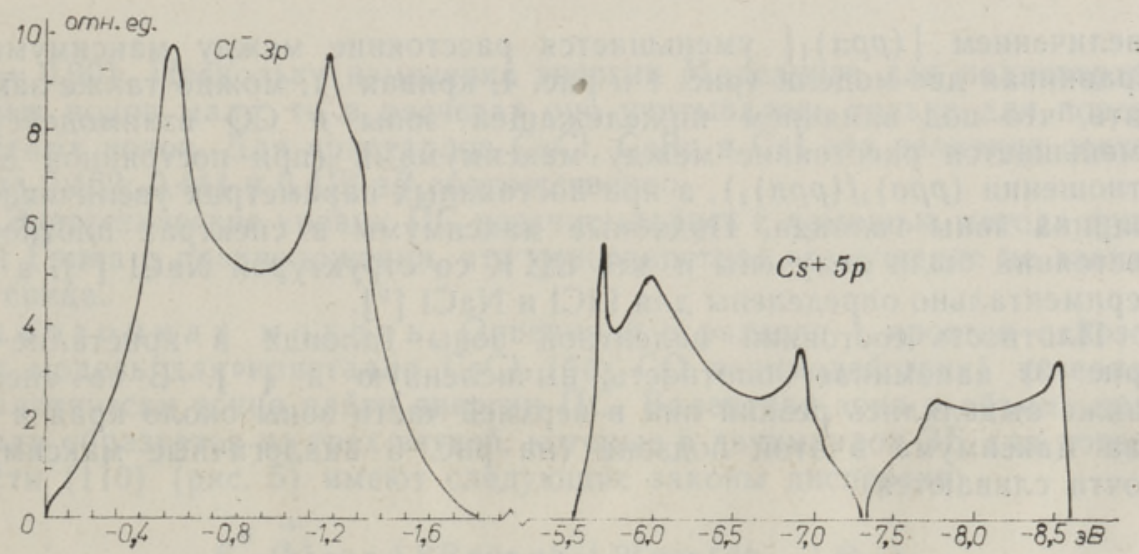

Рис. 1. Плотность состояний в валентных зонах $\mathrm{CsCl}$.

в центре ЗБ. В кристалле $\mathrm{CsCl}$ минимум энергии в $5 p$-зоне $\mathrm{Cs}^{+}$приходится на точку $M$, а в остальных зонах - на точку $R$. Значения энергий в симметричных точках $\Gamma, X, R$ приведены в табл. 2.

На основе вычисленных значений энергий получены плотности состояний валентных зон (рис. $1-3$ ). Энергетическая структура $5 p$-зоны $\mathrm{Cs}^{+}$мало отличается в разных кристаллах, поэтому она показана только для $\mathrm{CsCl}$.

Резко выделенный максимум плотности состояний в нижней части зоны галоида в кристаллах $\mathrm{CsBr}$ и CsI объясняется CO взаимодействием, а остальные максимумы в этих зонах, как и два максимумаз в $3 p$-зоне $\mathrm{Cl}^{-}$, обусловлены выбором значений резонансных интегралов. Влияние последних на вид спектра видно из рис. 4, где показаны плотности состояний в предельно простой модели кристалла $\mathrm{CsCl}$, в которой возможны только взаимодействия между ближайшими соседями $\mathrm{Cl}^{-}$ (без СО взаимодействия). Видно, что при постоянной ширине зоны с 


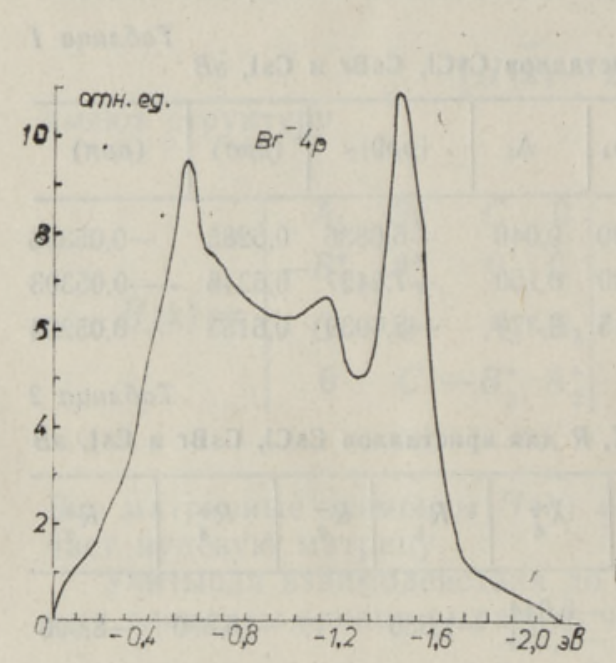

Рнс. 2. Плотность состояний в валентной зоне $\mathrm{CsBr}$.

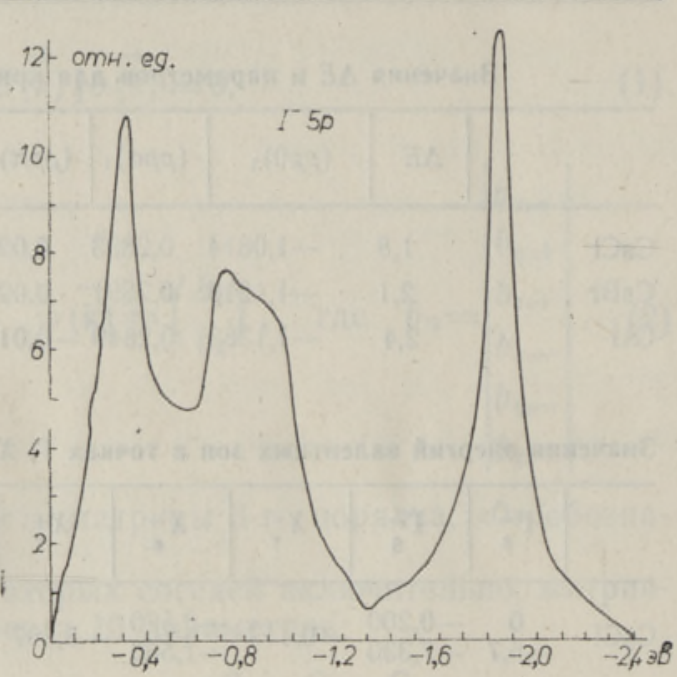

Рис. 3. Плотность состояний в валентной зоне CsI

увеличением $\left|(p p \pi)_{1}\right|$ уменьшается расстояние между максимумами. Сравнивая две модели (рис. 1 и рис. 4, кривая 1), можно также заключить, что под влиянием нижележащей зоны и СО взаимодействия уменьшается расстояние между максимумами (при постоянной $\Delta E$ и отношении $\left.(p p \sigma)_{1} /(p p \pi)_{1}\right)$, а при постоянных параметрах увеличивается ширина зоны галоида. Подобные максимумы в спектрах плотностей состояний были получены и для ЩГК со структурой $\mathrm{NaCl}\left[{ }^{4}\right]$, а экспериментально определены для $\mathrm{LiCl}$ и $\mathrm{NaCl}\left[{ }^{9}\right]$.

Плотность состояний валентной зоны галоида в кристалле CsI (рис. 3) напоминает плотность, вычисленную в $\left[{ }^{10}\right]$. В еe спектре также выделялись резкий пик в верхней части зоны около края и еще два максимума в этой подзоне (на рис. 3 аналогичные максимумы почти сливаются).

\section{2. Электронная структура полуограниченного кристалла}

Расчеты [11] показывают, что ноны на поверхности (110) кристаллов $\mathrm{CsCl}, \mathrm{CsBr}$ и CsI испытывают перпендикулярные к поверхности сдвиги того же порядка, что ионы на поверхности (100) ЩГК со структурой $\mathrm{NaCl}$. Поскольку эти сдвиги очень малы (сотые доли постоянной решетки) и реконструкция чистых поверхностей ЩГК экспериментально не обнаружена, то здесь рассматривались только идеальные поверхности без изменения конфигурации ионов.

Полуограниченные кристаллы моделировались, как и в $\left[{ }^{4}\right]$, путем обрывания взаимодействия между ионами, расположенными по разные стороны от разрезающей плоскости. Известно, что в такой модели возможны только ПС типа Шокли. Для реального ионного кристалла необходимо еще учитывать изменение потенциала на поверхности, основной вклад в который дает изменение энергии Маделунга. С помощью метода Эвьена рассчитывались константы Маделунга для ионов на поверхности (110) и на следующем слое. Получены следующие значения: $\alpha_{s}=$ $=1,64935, \quad \alpha_{s s}=1,7684 \quad\left(\alpha_{b}=1,76267\right), \quad \gamma_{s} \equiv \alpha_{s} / \alpha_{b}=0,9357 \quad$ (в $\left[{ }^{2}\right]$ 


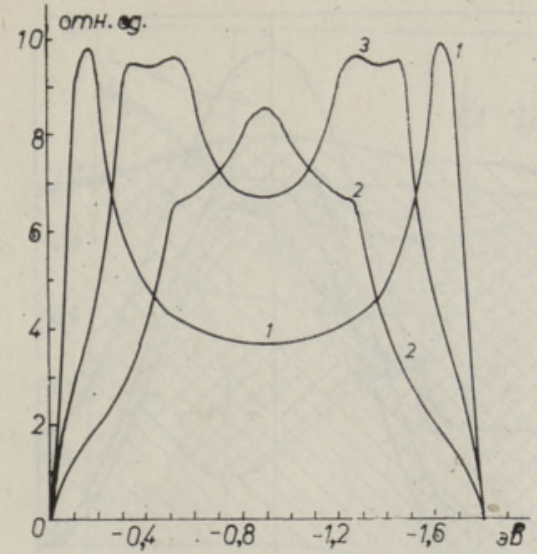

Рис. 4. Плотность состояний в валентной зоне однозонной модели $\mathrm{CsCl}$ : $1-(p p \sigma)_{1}=0,3953, \quad(p p \pi)_{1}=$ $=0,0273{ }_{\Im} B ; 2-(p p \sigma)_{1}=0,200$, $(p p \pi)_{1}=0,125{ }^{\circ} B ; 3-(p p \sigma)_{1}=$ $=0,300,(p p \pi)_{1}=-0,075{ }_{3} B$.

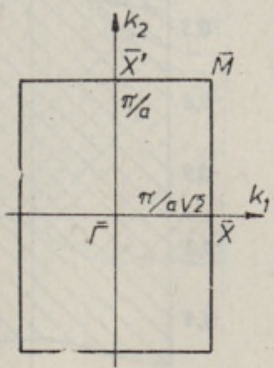

Рис. 5. Зона Бриллюэна для поверхности (110) простой кубической решетки.

$\left.\gamma_{s}=0,90\right)$. Поскольку изменение энергии Маделунга для подповерхностных ионов мало, то в расчетах оно учитывалось только для поверхностных ионов. Для кристаллов $\mathrm{CsCl}, \mathrm{CsBr}$ и $\mathrm{CsI}$ эта величина составляла 0,$462 ; 0,444$ и 0,419 эB соответственно.

Энергетические уровни ПС рассчитывались с помощью метода функций Грина в предположении, что поверхностное возмущение не завиєит от спина.

Однозонная модель. Описанная в разделе 1 простая однозонная модель для кристалла $\mathrm{CsCl}$ (без $\mathrm{CO}$ взаимодействия) позволяет аналитически точно найти энергии ПС. Валентная зона в объеме кристалла образуется из трех ветвей, которые в двухмерной ЗБ для поверхности (110) (рис. 5) имеют следующие законы дисперсии:

$$
\begin{aligned}
& \left.E_{1,2}^{0} \overrightarrow{(\mathbf{k}}\right)=\varepsilon+2 B \cos a k_{2}+2 f \cos \left(d k_{\perp}+\Theta_{1,2}\right), \\
& \left.E_{3}^{0} \overrightarrow{(\mathbf{k}}\right)=\varepsilon+2 A \cos a k_{2}+4 B \cos d k_{1} \cos d k_{\perp},
\end{aligned}
$$

где $\varepsilon=(p p 0)_{1}, \quad A=(p p \sigma)_{1}, \quad B=(p p \pi)_{1}, \quad f \mathrm{e}^{i \Theta_{1}}=A \mathrm{e}^{\mathrm{l} d k_{1}}+B \mathrm{e}^{-\mathrm{i} d \mathbf{k}_{1}}, \quad f^{2}=$ $=A^{2}+B^{2}+2 A B \cos \sqrt{2} a k_{1}, \Theta_{2}=-\Theta_{1}, k_{\perp}-$ компонента волнового вектора $\overrightarrow{\mathbf{k}}$, перпендикулярная к поверхности $\left(\left|k_{\perp}\right| d \leqslant \pi\right), d=a / \sqrt{2}$.

Поскольку $E_{1}{ }^{0}\left(k_{1}, k_{2}\right)=E_{2}{ }^{0}\left(k_{1}, k_{2}\right)$, то ПС, отщепляющиеся от этих ветвей, двукратно вырождены

$$
E_{1,2}=\varepsilon+2 B \cos a k_{2}+\Delta \varepsilon^{\prime}+f^{2} / \Delta \varepsilon^{\prime},
$$

с условием возникновения $\Delta \varepsilon^{\prime}>f$. Третий уровень с энергией

$$
E_{3}=\varepsilon+2 A \cos a k_{2}+\Delta \varepsilon^{\prime \prime}+\left(2 B \cos d k_{1}\right)^{2} / \Delta \varepsilon^{\prime \prime}
$$

возникает при условии

$$
\Delta \varepsilon^{\prime \prime}>2 B \cos d k_{1}
$$




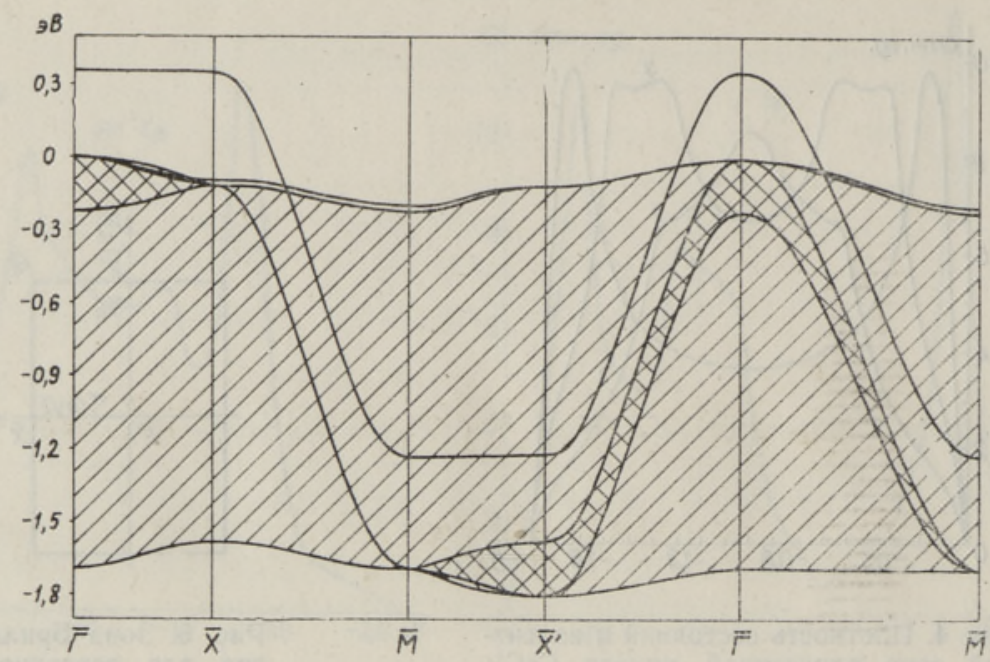

Рис. 6. Энергетические уровни в области валентной зоны на поверхности (110) однозонной модели $\mathrm{CsCl}$. Заштрихованные участки непрерывный спектр (здесь и на следующих рисунках).

Здесь $\Delta \varepsilon^{\prime}$ и $\Delta \varepsilon^{\prime \prime}$ обозначают изменение кулоновских интегралов поверхностных ионов для $p$-гантелей, образующих угол в $45^{\circ}$ с нормалью к поверхности, и для $p$-гантели с осью, параллельной поверхности.

Из соотношения (6) видно, что третий уровень ПС появляется при любых параметрах $\left(\Delta \varepsilon^{\prime \prime} \neq 0\right)$ в направлении $\bar{X}-\bar{M}$. На рис. 6 для симметричных направлений в двухмерной ЗБ нанесены проекции объемных энергий и поверхностные уровни при параметрах $\varepsilon=-0,9, B=0,0273$, $A=0,3953, \Delta \varepsilon^{\prime}=\Delta \varepsilon^{\prime \prime}=0,462$ эВ. Энергии связи ПС в точке $\bar{\Gamma}$ равны 0,0034 (двукратно вырожденный уровень) и 0,359 эВ. В отличие от $\left[{ }^{12}\right]$, где рассматривалась поверхность (100) кристаллов типа $\mathrm{NaCl}$, на поверхности (110) $\mathrm{CsCl}$ в одном узле только одна $p$-гантель параллельна поверхности и поэтому ниже по энергии расположен двукратно вырожденный уровень. Последнее утверждение справедливо лишь при не очень большом соотношении $\Delta \varepsilon^{\prime} / \Delta \varepsilon^{\prime \prime}$, предельное значение которого зависит от соотношения интегралов $A$ и $B$, а также от энергии ПС (в точке $\bar{\Gamma}$ на границе объемных зон $\Delta \varepsilon_{\text {mp }}^{\prime}=((A+B) / 2 B) \Delta \varepsilon^{\prime \prime}=7,73 \Delta \varepsilon^{\prime \prime}$, а при $\Delta \varepsilon^{\prime \prime}=0,462$ эв $\left.\Delta \varepsilon_{\text {пр }}^{\prime}=2,23 \Delta \varepsilon^{\prime \prime}\right)$. Различием является также то, что в $\mathrm{CsCl}$ двукратное вырождение сохраняется во всей двухмерной ЗБ, а третий локальный уровень ПС простирается глубоко в валентную зону (рис. 6).

Д вухзонная модель. В рамках двухзонной модели, описанной в разделе 1, вычисленные энергии бесконечного кристалла $\mathrm{CsCl}$ в двухмерной ЗБ показывают (рис. 7) увеличение зависимости от волнового вектора и появление участков запрещенных энергий в области валентной зоны.

Учет нижележащей p-зоны и СО взаимодействия значительно усложняет задачу о нахождении энергий ПС. Разрезая кристалл плоскостью $(\overline{22} 0)$ и следуя общей схеме метода функций Грина, компоненту волновой функции $C_{l}^{\alpha}$, перпендикулярную к поверхности, и энергии ПС можно найти из системы уравнений

$$
C_{l^{\prime}}^{\alpha}=\sum_{n=1,2} \sum_{\substack{v=+,-l=0,1}}\left\{g _ { l ^ { \prime } l } ^ { \alpha x _ { n } v } \left[\Delta \varepsilon_{n}^{\prime} C_{l^{n}}^{x_{n} v}-D_{n} C_{v}^{x_{n} v}-P C_{v^{m}}^{x_{m} v}+\right.\right.
$$




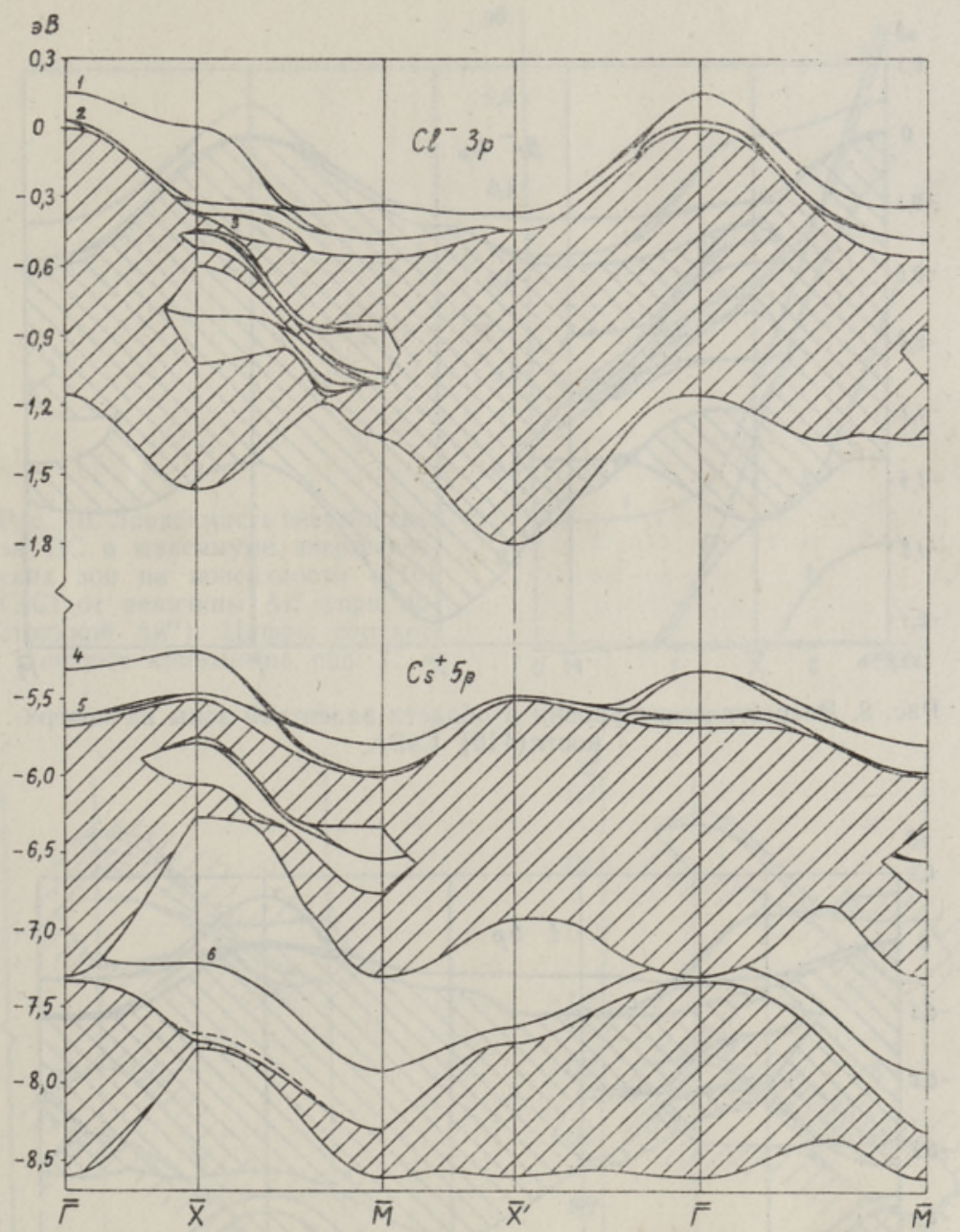

Рнс. 7. Энергетические уровни в области валентных зон на поверхности (110) двухзонной модели $\mathrm{CsCl}$.

где

$$
\begin{aligned}
& \left.+T C_{\gamma}^{y_{m} v} \cos \left(a k_{2} / 2\right)-R C_{\gamma}^{z_{m} v}\right]+g_{l^{\prime} l}^{\alpha y_{n} v}\left[\Delta \varepsilon_{n}^{\prime} C_{l}^{y_{n} v}-D_{n}^{*} C \underset{\gamma}{y_{n} v}-\right. \\
& \left.-P C_{\gamma}^{y_{m} v}+T C_{\gamma}^{x_{m} v} \cos \left(a k_{2} / 2\right)+R C_{\gamma}^{z_{m} v}\right]+g_{l^{\prime} l}^{\alpha z_{n} v}\left[\Delta \varepsilon_{n}^{\prime \prime} C_{l}^{z_{n} v}-\right.
\end{aligned}
$$

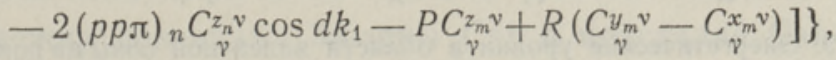

$$
m=n-(-1)^{n}, \quad \gamma=l+(-1)^{l},
$$

$D_{n}=\left[(p p \sigma)_{n}+(p p \pi)_{n}\right] \cos d k_{1}+\mathrm{i}(-1)^{l}\left[(p p \sigma)_{n}-(p p \pi)_{n}\right] \sin d k_{1}$, $P=(2 / 3)[(p p \sigma)+2(p p \pi)] \cos \left(a k_{2} / 2\right), \quad R=\mathrm{i}(-1)^{l} T \sin \left(a k_{2} / 2\right)$, $T=(2 / 3)[(p p \sigma)-(p p \pi)], \quad g_{l^{\prime}}^{\alpha \beta}=N^{-1 / 3} \sum_{k_{\perp}}^{\Sigma} \frac{b_{\alpha}(\overrightarrow{\mathbf{k}}) b_{\beta}^{*}(\overrightarrow{\mathbf{k}})}{E-E(\overrightarrow{\mathbf{k}})} \exp \left\{\mathrm{i} d k_{\perp}\left(l-l^{\prime}\right)\right\}$. 


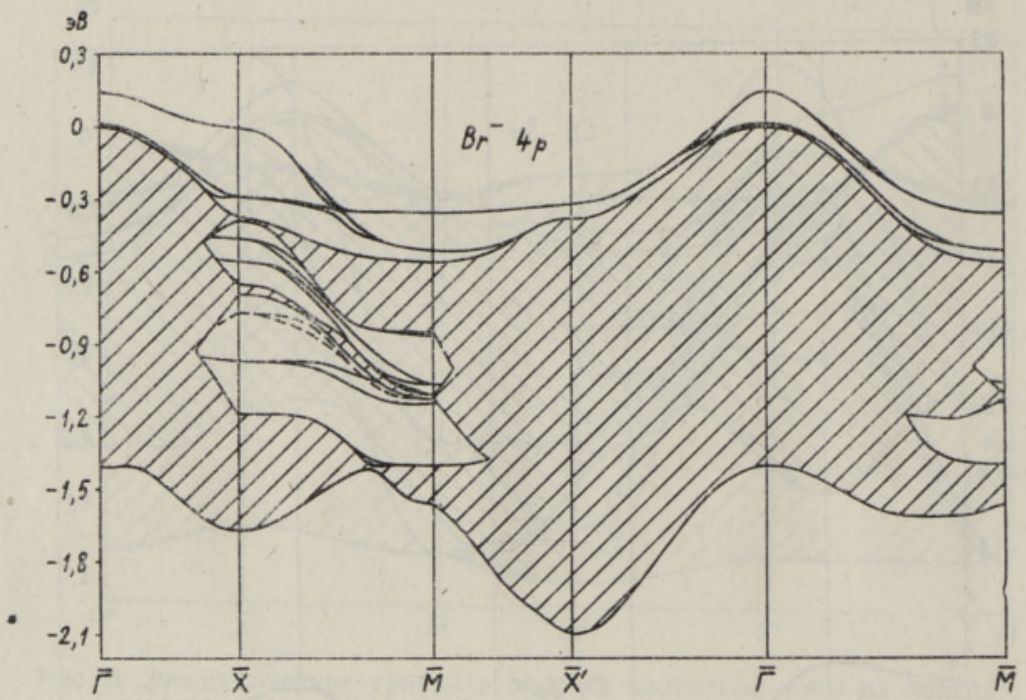

Рис. 8. Энергетические уровни в области валентной зоны на поверхности (110) $\mathrm{CsBr}$.

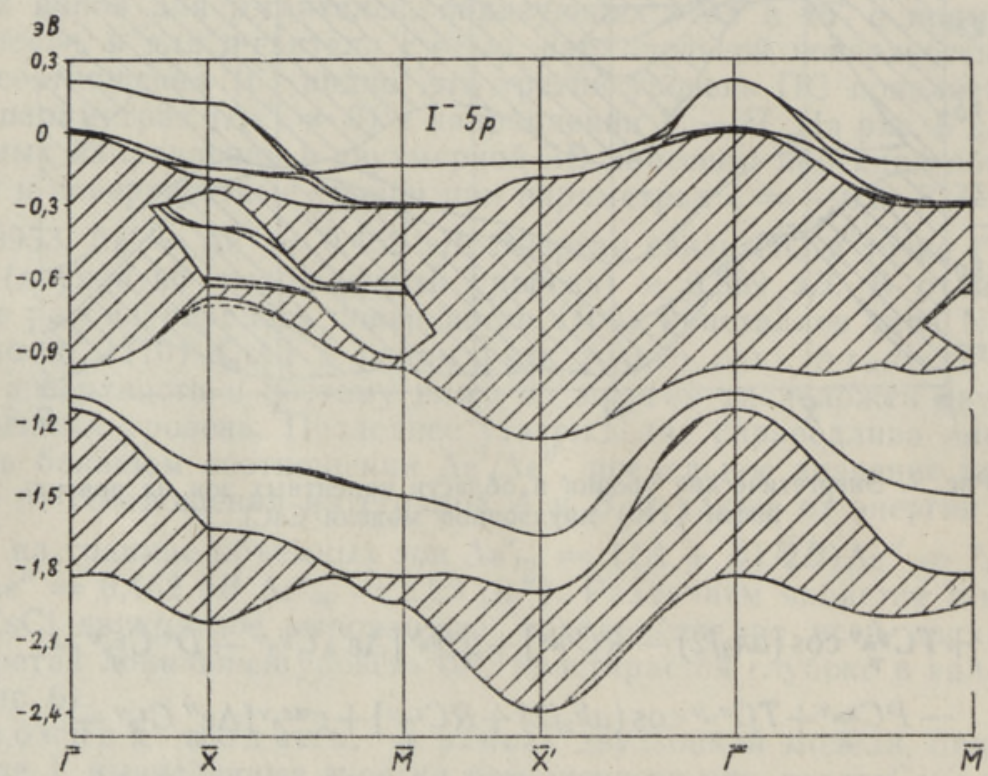

Рис. 9. Энергетические уровни в области валентной зоны на поверхности (110) CsI.

Индекс $l^{\prime}$ принимает значения 0 и $1 ; \quad u-x_{p^{\prime}} v^{\prime}, y_{p} v^{\prime}, z_{p} v^{\prime}\left(v^{\prime}=\right.$ $=+,-; p=1,2)$.

Вычисленные энергетические уровни при $\Delta \varepsilon_{n}^{\prime}=\Delta \varepsilon^{\prime \prime}{ }_{n}=\Delta \varepsilon$ показаны на рис. 7-9 в симметричных направлениях двухмерной ЗБ. Для всех кристаллов характерно появление ПС выше объемных зон и в щелях запрещенной энергии. На рис. 8, 9 энергии в области $5 p$-зоны $\mathrm{Cs}^{+}$не показаны, поскольку энергетическая структура там весьма 
Pнс. 10. Зависимость энергии связи ПС в максимуме энергетических зон на поверхности (110) $\mathrm{CsCl}$ от величнны $\Delta \varepsilon^{\prime}$ (при постоянной $\left.\Delta \varepsilon^{\prime \prime}\right)$. Цифры соответствуют кривым на рис. 7.
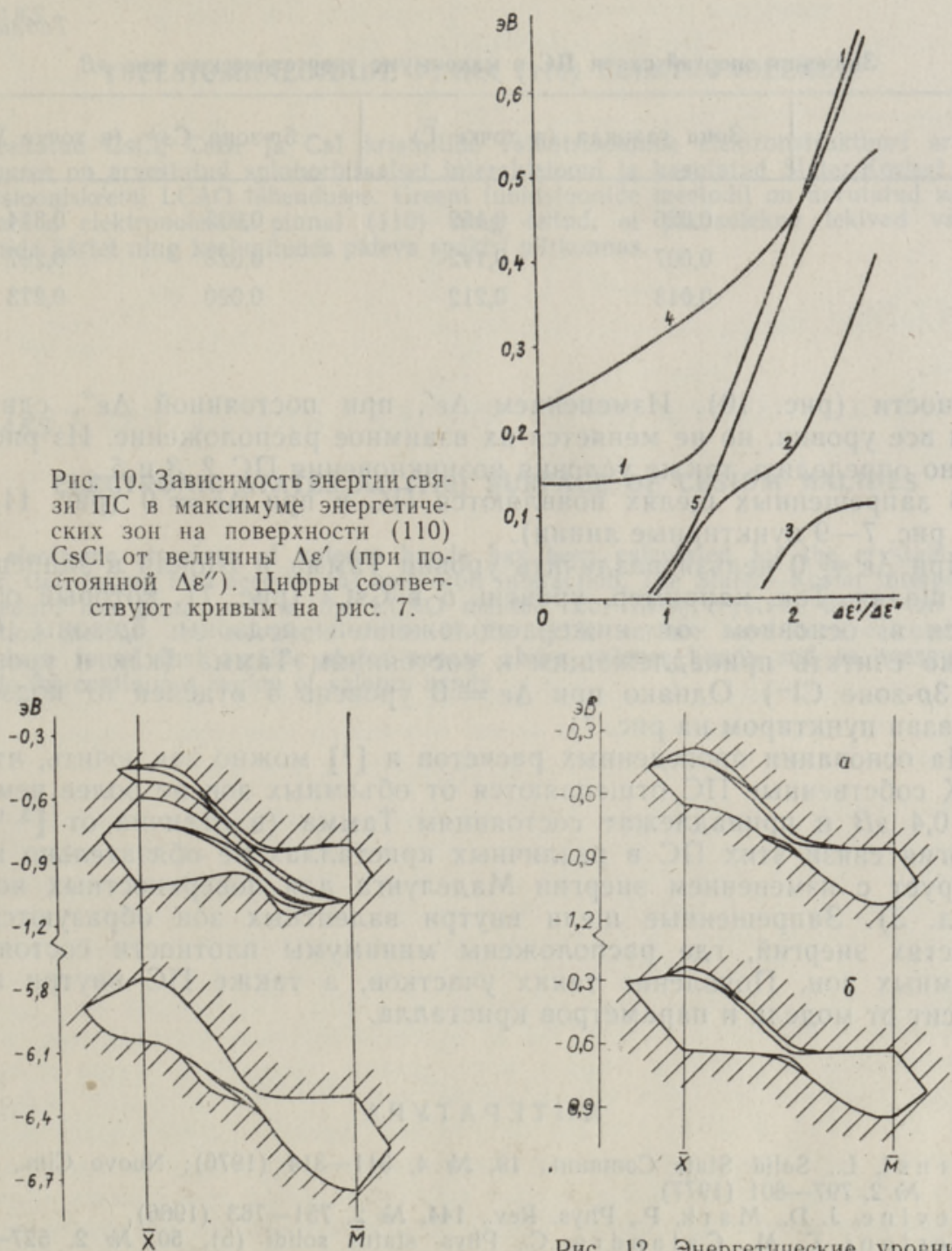

Рис. 11. Энергетические уровни ПС в запрещенных щелях валентных зон $\mathrm{CsCl}$ при $\Delta \varepsilon=0$.

Рис. 12. Энергетические уровни ПС в запрещенной щели валентной зоны при $\Delta \varepsilon=0: \operatorname{CsBr}(a)$, CsI (б),

сходна в различных кристаллах. Можно отметить лишь появление ПС в точке $\Gamma$ между подзонами $5 p$-зоны $\mathrm{Cs}^{+}$в кристалле CsI (при энергии $-9,19$ эВ).

Под влиянием СО взанмодействня вырождение снимается, но образующийся третий уровень ПС выше объемных зон не появляется (за исключением направления $\bar{X}-\bar{M}$ в $\mathrm{CsCl}$ ). Энергии связи остальных ветвей в максимуме зон (табл. 3) больше отличаются между собой, чем в ЩГК типа $\mathrm{NaCl}\left[{ }^{4}\right]$. Это обусловлено более широкими объемными зонами в данной модели.

Существенным различием (по сравнению с $\left[{ }^{4}\right]$ ) является зависимость энергий связи ПС от величины изменения кулоновского интеграла $\Delta \varepsilon^{\prime}$ для $p_{x, y}$-гантелей, образующих угол в $45^{\circ}$ с нормалью к по- 
Значения энергий связи ПС в максимуме энергетических зон, эB

\begin{tabular}{l|cc|cc}
\hline & Зона галоида $($ в точке $\bar{\Gamma})$ & $5 p$-зона $\mathrm{Cs}^{+}$(в точке $\bar{X}$ ) \\
\hline $\mathrm{CsCl}$ & 0,026 & 0,152 & 0,035 & 0,314 \\
$\mathrm{CsBr}$ & 0,007 & 0,142 & 0,028 & 0,297 \\
$\mathrm{CsI}$ & 0,013 & 0,212 & 0,020 & 0,273
\end{tabular}

верхности (рис. 10). Изменением $\Delta \varepsilon^{\prime}$, при постоянной $\Delta \varepsilon^{\prime \prime}$, сдвигаются все уровни, но не меняется их взаимное расположение. Из рис. 10 можно определить также условия возникновения ПС 2, 3 и 5 .

В запрещенных щелях появляются ПС и при $\Delta \varepsilon=0$ (рис. 11,12 и на рис. $7-9$ пунктирные линии).

При $\Delta \varepsilon \neq 0$ нельзя различить уровни Тамма и Шокли в запрещенных щелях. Так, например, уровень 6 в $\mathrm{CsCl}$ (рис. 7), который образуется в основном от нижерасположенной подзоны $5 p$-зоны $\mathrm{Cs}^{+}$, можно считать принадлежащим к состояниям Тамма (как и уровень 3 в $3 p$-зоне $\left.\mathrm{Cl}^{-}\right)$. Однако при $\Delta \varepsilon=0$ уровень 6 отделен от подзоны (показан пунктиром на рис. 7).

На основании проведенных расчетов и $\left[{ }^{4}\right]$ можно заключить, что в ЩГК собственные ПС отщепляются от объемных зон не более чем на $0,3-0,4$ эB и принадлежат состояниям Тамма (в отличие от $[3,13]$ ). Энергия связи этих ПС в различных кристаллах не обязательно коррелирует с изменением энергии Маделунга для поверхностных ионов (табл. 3). Запрещенные щели внутри валентных зон образуются в областях энергий, где расположены минимумы плотности состояний объемных зон. Появление таких участков, а также ПС внутри них, зависит от модели и параметров кристалла.

\section{Л И ТЕРА Т Р А}

1. Ernst, L., Solid State Commun., 19, № 4, 311-314 (1976); Nuovo Cim., 39B, № 2, 797-801 (1977).

2. Levine, J. D., Mark, P., Phys. Rev., 144, № 2, 751-763 (1966).

3. Bertoni, C. M., Calandra, C., Phys. status solidi (b), 50, № 2, 527-536 (1972).

4. С а к с Т., З а в т Г., Изв. АН ЭССР, Физ. Матем., 27, № 2, 174-183 (1978).

5. O nodera, Y., J. Phys. Soc. Jap., 25, № 2, 469-480 (1968).

6. R ös s le r, U., Phys. status solidi, 34, № 1, 207-212 (1969).

7. Gout, C., Jouanin, C., Albert, J.-P., D a ude, N., C. r. Acad. sci. B., 278, № $1,23-24$ (1974).

8. S m it h, J. A., P o n g, W., Phys. Rev., B12, № 12, 5931-5936 (1975).

9. P on g, W., Smith, J. A., Phys. Rev., B9, № 6, 2674-2678 (1974).

10. Di Stefano, T. H., Phys. Rev., B7, № 4, 1564-1571 (1973).

11. B e n s o n, G. C., J. Chem. Phys., 35, № 6, 2113-2116 (1961).

12. З а в т Г. С., С а к с Т. Я., Физ. твердого тела, 14, вып. 10, 2897-2901 (1972).

13. Ven-Chung Lee, How - S en Wong, J. Phys. Soc. Jap., 45, № 3, 895-898 (1978). 
T. SAKS

\section{TSEESIUMHALOIDIDE PINNA (110) ELEKTRONOLEKUD}

On esitatud $\mathrm{CsCl}, \mathrm{CsBr}$ ja $\mathrm{CsI}$ kristallide valentstsoonide elektronstruktuuri arvutus, kusjuures on arvestatud spinnorbitaalset interaktsiooni ja kasutatud Slater-Kosteri interpolatsiooniskeemi LCAO lähenduses. Greeni funktsioonide meetodil on arvutatud samade kristallide elektronolekud pinnal (110) ning leitud, et pinnaolekud tekivad valentstsoonide äärtel ning keelupiludes pideva spektri piirkonnas.

\section{T. SAKS}

\section{ELECTRONIC STATES AT (110) SURFACE OF CESIUM HALIDES}

The electronic structure of valence bands has been calculated for the crystals $\mathrm{CsCl}$, $\mathrm{CsBr}$, CsI, taking into account the spin-orbit interaction. The Slater-Koster interpolation procedure has been used in the LCAO method. For these crystals, using the Green function method, the electronic states at the (110) surface have been calculated. It has been found that surface states appear above valence bands and in energy gaps inside the continuous region of valence bands. 